\title{
5 ADHS und KISS-/KIDD-Syndrom
}

Johannes Buchmann

\begin{abstract}
ADHS-Kinder („Aufmerksamkeits-Defizit-Hyperaktivitäts-Syndrom“) sind selten nur hinsichtlich ihrer drei Kardinalsymptome gestört. Fein- und grobmotorische Ungeschicklichkeit, fehlendes Gefühl für die eigene Motorik und beim primär aufmerksamkeitsgestörten Typ des ADHS (so genanntes Aufmerksamkeitsdefizitsyndrom „ADS“) der eingeschränkte Antrieb zur Bewegung sind motorische Symptome, die viele Eltern bemerken. Nicht umsonst werden derartige Auffälligkeiten in der ICD-1o als mögliche Begleitsymptome des hyperkinetischen Syndroms (HKS, F go.o und F 90.1) aufgeführt. Diese Kinder werden Ärzten, Physiotherapeuten, Ergotherapeuten, Osteopathen und anderen in der Einschätzung von motorischen Leistungen Geschulten primär oft wegen der auffälligen Motorik vorgestellt.

Im Säuglingsalter finden sich dazu ebenfalls viele Kinder mit einer leicht verlangsamten motorischen Entwicklung, weiter die „Schrei- und Spuckkinder" und Kinder, die eine so genannte „C-Skoliose“ ausbilden (s. Abb. 6). Damit ist eine bevorzugte Kopfhaltung in Seitneige und/oder Rotation gemeint, die in Rückenlage von einer Beckenauslenkung zu derselben Seite begleitet wird.

Es ist der unbestreitbare Verdienst von H. Biedermann, diese Vorzugshaltung beschrieben $\mathrm{zu}$ haben als verursacht durch eine manualmedizinische Funktionsstörung der Kopfgelenke (Biedermann 1991; Biedermann 1993; Biedermann 1995; Biedermann 1995), wenn derartige Zusammenhänge auch schon länger vermutet wurden (Mau 1962; Gutmann 1968; Mau 1979; Riede and Tomaschewski 1983). Diese Funktionsstörung ist aufzufassen als eine segmentale Dysfunktion, die prinzipiell auf der Crundlage gestörter propriozeptiver Afferenzen hauptsächlich aus den Gelenkkapseln der kleinen Wirbelge-
\end{abstract}


lenke des betroffenen Segmentes und den zugehörigen myofaszialen Strukturen entsteht (Speckmann and Wittkowski 1997; Buchmann, Wende et al. 2005). Im Kopfgelenksbereich (Segmente $\mathrm{O} / \mathrm{C}_{1}, \mathrm{C}_{1} / \mathrm{C}_{2}$ und $\mathrm{C}_{2} / \mathrm{C}_{3}$ ) kommen propriozeptive Afferenzen aus den Ligg. alaria und aus den kleinen Nackenmuskeln hinzu. Das führt beim Säugling bezüglich der spontanen Kopfstellung zur Bevorzugung einer Seite, die aber als „Vermeidung“ der Gegenseite entsteht. Reflektorisch kommt es dann zu der bereits erwähnten Beckenauslenkung zu derselben Seite der primären spontanen Kopfstellung. Biedermann nannte das eine „kopfgelenkinduzierte Symmetriestörung“ (KISS) (Biedermann 1991). Nach einer manualtherapeutischen Behandlung solle sich diese Symmetriestörung auflösen. Als Ursache dieses Syndroms werden meist perinatale Traumata angeführt (Mau 1979; Biedermann 1995; Biedermann 1995).

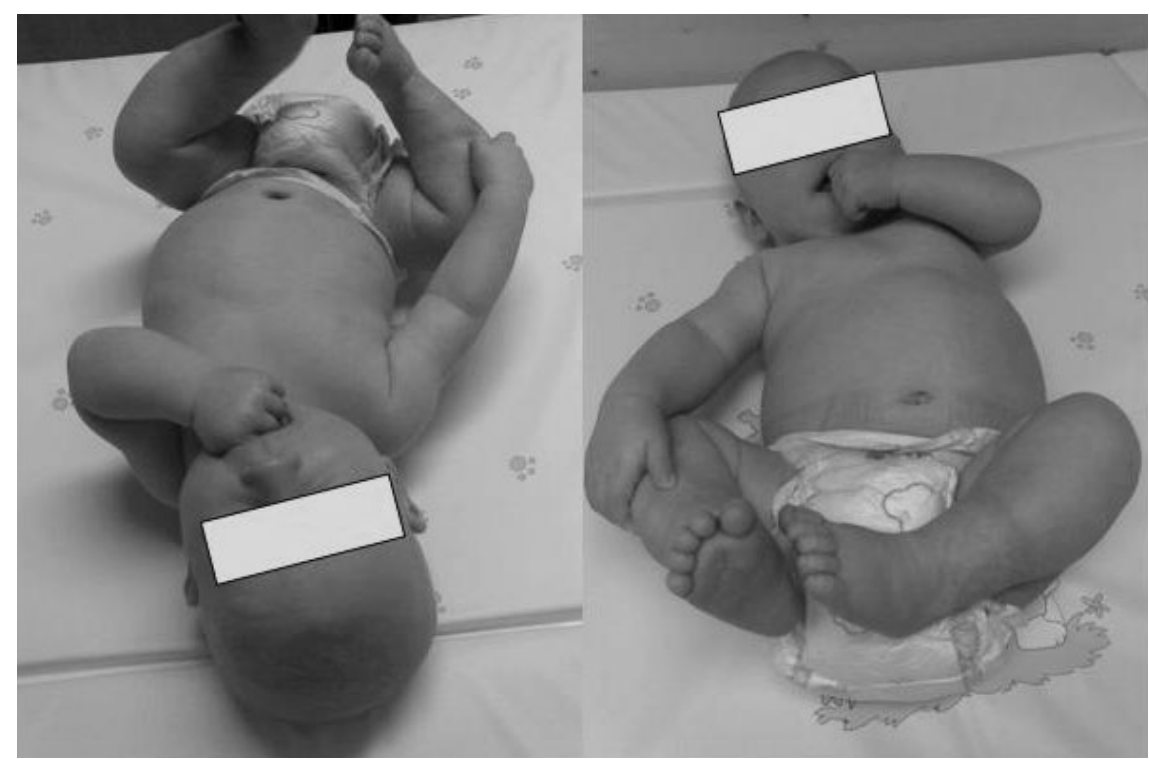

Abb. 6 C-Skoliose

Das primäre KISS-Syndrom, oft „KISS-I genannt“, ist motorisch also gekennzeichnet durch:

- spontane Kopfstellung in Seitneige und/oder Rotation

- Beckenauslenkung zu derselben Seite.

Manualmedizinisch findet sich meist eine segmentale Dysfunktion $\mathrm{C}_{1} / \mathrm{C}_{2}$ und $\mathrm{C}_{2} / \mathrm{C}_{3}$, oft verbunden mit einer thorakolumbalen Funktionsstörung und einer Funktionsstörung der Sakroiliakalgelenke. Es ist dabei zu bedenken, dass solche segmentalen Lateralisationszustände physiologisch bei Säuglingen passager nachweisbar sind (Seifert 1981; Buchmann and Bülow 1983; Buchmann, Bülow et al. 1992). Weshalb sich bei Einigen diese „verfestigen“ und dann als KISS-Syndrom imponieren, ist nicht sicher bekannt (Biedermann 1995; Bie- 
dermann 1995). Es ist aber unschwer vorstellbar, dass auf dieser Grundlage die Kinder sich verzögert und nur über eine Seite drehen und auch ein seitendifferentes Krabbeln aufweisen. Der oft gesehene Zusammenhang von Kopfgelenkstörung und „Schreikind“ außerhalb der „Dreimonatskoliken“ ist eventuell ähnlich aufzufassen, als Reaktion des Kindes auf eingeschränkte, eventuell sogar schmerzhafte Kopfdrehung bzw. Seitneige. Direkte empirische Beweise für diese klinischen Beobachtungen stehen jedoch noch aus.

An weiteren nachvollziehbaren Symptomen des KISS-Syndroms im Säuglingsalter sind zu nennen:

- seitenbetonter Haarabrieb occipital,

- leichte (!) Schädeldeformationen durch die Vorzugshaltung auch beim Schlafen,

- einseitige Stillprobleme, da die Kinder den Kopf zur gestörten Seite nicht gerne drehen bzw. neigen (Biedermann 1993).

Zusammengefasst werden die letzten Symptome auch als „KISS-II“ Syndrom bezeichnet.

In der Laienpresse, einigen Publikationen (Biedermann and Koch 1996; Theiler 1996) und auch auf einer Vielzahl von Internetseiten (s. z. B. www. kiss-kiddsyndrom.com) tauchen dann unabhängig vom Alter noch weitere „KISS-Symptome“ auf, die jedoch auf der Crundlage einer segmentalen Dysfunktion der Kopfgelenke nicht zu erklären sind. Dazu zählen Gesichtsschädelasymmetrien und Hüftreifungsstörungen, Sichel- und Spitzfüsse, Durchfall und Verstopfung, Tonusanomalien und ausgeprägte opisthotone Schlafhaltungen, unerklärliches Sozialverhalten, Konzentrationsstörungen, Kopfschmerzen bis zur Migräne und sogar motorische und vocale Tic's (Tourette Syndrom). Zusammen mit dann im Schulalter auftetenden Lern- und Verhaltensstörungen werden sie auch als „KIDD-Syndrom “ bezeichnet. KIDD bedeutet „kopfgelenkinduzierte Dysgnosie/Dyspraxie“. Dysgnosie sei eine Störung des Formerkennungsvermögens, Dyspraxie meint die motorische Ungeschicklichkeit. Diese Kinder hätten Koordinationsstörungen und „Wahrnehmungsstörungen“. Der letzte Begriff taucht sehr häufig im Umfeld des KISS/KIDDSyndroms auf. Er ist jedoch völlig unscharf definiert. Verstanden wird darunter meist eine generelle Störung propriozeptiver, motorischer, konzentrativer und exekutiver Funktionen sowie Verhaltensstörungen. Ätiopathogenetisch sei das „KIDD-Syndrom“ die Folge eines unbehandelten „KISS-Syndroms“. Zum „KIDD-Syndrom“ werden folgende Symptome angeführt (Zusammenstellung):

- Kopfschmerzen, Migräne

- Haltungsschwächen

- Bewegungseinschränkungen

- motorische und vocale Tic's

- Koordinationsschwierigkeiten (Fahrradfahren, Balancieren)

- motorische Defizite

v Lern- und Konzentrationsstörungen in der Schule 
- Wahrnehmungsstörungen

- gestörte soziale Integration

- Emotionsstörungen: Frustration $\rightarrow$ Reizbarkeit $\rightarrow$ Ungeduld $\rightarrow$ Aggressivität

- Schreib-und Leseschwierigkeiten bishin zur Legasthenie und Dyskalkulie

- Lernstörungen.

Diese Symptomatik umfasst, abgesehen von den Kopfschmerzen und „Migräne“, den ICD-10 Bereich F 80-F 95 sowie die Lernbehinderung. Originäre Literatur oder empirische Forschung dazu ist in indizierter medizinischer Literatur nicht aufzufinden (Literaturrecherche „PubMed“ November 2007). Es ist allerdings nur schwer oder besser gar nicht erklärbar, weshalb eine simple manualmedizinische Funktionsstörung der Kopfgelenke zu einer solchen Vielzahl verschiedener Symptome führen sollte. Meist wird der Zusammenhang einer „Reaktion“ des betroffenen Kindes auf die nicht behandelte Kopfgelenkstörung postuliert. Die primäre Kopfgelenkstörung führe zu verzögerter motorischer Entwicklung und zu den bereits erwähnten „Konzentrations- bzw. Wahrnehmungsstörungen“ (Coenen 1996; Coenen 2002). Die Kinder kämen in der Schule nicht mit (Lernstörungen, Lese- und Rechtsschreibstörungen), würden ausgegrenzt und reagierten mit Verhaltensstörungen bis hin zum hyperkinetischen Syndrom. Hier schließt sich der Kreis zum ADHS. Zitat aus einer WebSite:

„Bei Konzentrationsstörungen, Ein- und Durchschlafstörungen oder eventueller Hyperaktivität sollte ein Blick auf die Wirbelsäule, insbesondere die Halswirbelsäule geworfen werden." (www.kiss-kiddsyndrom.com).

Aufgrund der motorischen Störungen würden die Kinder später vermehrt über Kopf-, Rücken- und Knieschmerzen klagen. Sie würden grobmotorisch ungeschickt sein, oft hinfallen und Gangauffälligkeiten aufweisen. Dem Arzt und Manualmediziner werden nach unseren Erfahrungen (Spezialsprechstunde „Neurologische und manualmedizinische Untersuchung und Behandlung von Kindern und Jugendlichen " im Rahmen einer KV-Ermächtigung an einer Universitätsklinik) Kinder mit einer solchen Störung unter verschiedenen Differenzialdiagnosen vorgestellt (s. Tab. 2).

Tab. 2 Überweisungsdiagnosen an den Manualmediziner bei ADHS

motorische Ungeschicklichkeit

„Haltungsschwäche“

so genanntes KISS-Syndrom (kopfgelenkinduzierte-Symmetrie-Störung) oder

so genanntes KIDD-Syndrom (kopfgelenkinduzierte-Dysgnosie und Dyspraxie)

sensomotorische Integrationsstörung

„Wahrnehmungsdefizit“

Aufmerksamkeitsdefizit-Syndrom (ADS) 
Die Schwierigkeit liegt in der Beurteilung einer komorbiden Störung (z. B. Kopfgelenksstörung und Legasthenie) versus eines vermuteten Kausalzusammenhanges (z. B. Kopfgelenksstörung bzw. Verkettungssyndrome und motorische Ungeschicklichkeit/ADHS). Empirische Daten zur Häufigkeit oder Ausprägung manualmedizinischer Funktionsstörungen bei ADHS-Kindern sind nicht allgemein zugänglich publiziert (MedLine Recherche 11/2007; Suchbegriffe „ADHD“ oder „attention-deficite hyperactivity disorder“ und „somatic dysfunction“).

Nun ist unbestreitbar, dass ADHS-Kinder motorische Defizite aufweisen können. Mittels der transkraniellen Magnetstimulation gelang es in den letzten Jahren, motorische Inhibitionsdefizite bei Kindern mit ADHS zu objektivieren. Die transkranielle Magnetstimulation (TMS) hat in den letzten Jahren zunehmend an Bedeutung in der neuropsychiatrischen Forschung gewonnen. Durch dieses Verfahren können einzelne Hirnareale gezielt nicht-invasiv stimuliert werden. Mittels TMS ist es u.a. möglich, verschiedene Aspekte der kortikalen motorischen Inhibition und Fazilitation, zusammengefasst kortikale motorische Exzitabilität, zu messen. Dazu werden unterschiedliche TMSParadigmen eingesetzt. Fest in der Klinik etabliert ist die Überprüfung der Funktionsfähigkeit der Pyramidenbahn ( $\mathrm{MEP}=$ motorisch evozierte Potenziale) einschließlich der zentral motorischen Leitungszeit. Mittels Doppelpulsverfahren (zwei definierte Magnetreize mit distinkten Interstimulusintervallen) werden intrakortikale Inhibition (ICI) und Fazilitation (ICF) gemessen. Moll et al. beschrieben im Jahre 2000 als erste eine defizitäre intrakortikale motorische Inhibition (Moll et al. 200o) bei Kindern mit ADHS. Unserer Arbeitsgruppe gelang es, bei ADHS-Kindern eine gestörte interkortikale Inhibition (interhemispherielle oder transcallosale Inhibition, auch ipsilaterale silent period) nachzuweisen (Buchmann et al. 2003; Buchmann et al. 2006). Ebenso gelang es, eine insgesamt gestörte intrakortikale Abfolge inhibitorischer und exzitatorischer Vorgänge im Motorkortex betroffener Kinder aufzuzeigen (Buchmann et al. 2007). Insofern kann von einer gestörten Motorik bei ADHSKindern ausgegangen werden.

Die häufigste Frage an den Arzt ist dann die der Beeinflussbarkeit der motorischen Ungeschicklichkeit oder einer „Haltungsschwäche“. Aber auch Kopfschmerzen oder andere Schmerzsyndrome, die mit dem Bewegungssystem zusammenhängen, führen zur Vorstellung beim manualmedizinisch tätigen Pädiater/Hausarzt, Kinderneuropsychiater oder Orthopäden. Oftmals wird jedoch die Differenzialdiagnose nicht wirklich bedacht. In unserer eigenen Sprechstunde verbargen sich z. B. hinter der Überweisungsdiagnose „ADHS“ unter anderem die in Tabelle 3 aufgeführten Erkrankungen.

Die Schwierigkeit liegt wie bereits erwähnt in der Beurteilung von Komorbiditäten des ADHS versus motorische Störungen innerhalb des ADHS.

In einer eigenen, allerdings durch die geringe Fallzahl limitierten, Untersuchung konnten wir zeigen, dass sich bei ADHS-Kindern, die nachweisbare transcallosale Überleitstörungen aufwiesen, manualmedizinische Funktionsstörungen der Halswirbelsäule fanden (Buchmann and Häßler 2004). Die Be- 
handlung dieser segmentalen Funktionsstörungen hatte jedoch keinerlei Effekte auf die Ausprägung der Symptomatik des ADHS. Da etwa die Hälfte der untersuchten Kinder Kopfgelenkdysfunktionen aufwies, ist zu vermuten, dass aufgrund der hohen Prävalenz des ADHS (August et al. 1996; Rohde et al. 1999; Wender 2002) und der wahrscheinlich auch recht hohen Prävalenz manualmedizinischer Funktionsstörungen bei diesen Kindern es sich um reine Koinzidenzen handelt (Buchmann and Häßler 2004).

Tab. 3 Differenzialdiagnose des ADHS mit motorischen Auffälligkeiten

\begin{tabular}{l}
\hline Störung des Sozialverhaltens \\
\hline depressive Störung im Kindesalter \\
\hline emotionale Bindungsstörung \\
\hline Legasthenie, Dyskalkulie \\
\hline Lernbehinderung \\
\hline Artikulationsstörungen \\
\hline Tumoren der hinteren Schädelgrube \\
\hline Anlagestörungen Skelettsystem und zerebral \\
\hline altes, perinatal entstandenes Hämatom im M. sternocleidomastoideus \\
\hline zervikale Dystonie \\
\hline
\end{tabular}

Es ließe sich jedoch diskutieren, dass es aufgrund der „psychischen Erkrankung ADHS “ auch zu einer vermehrten generellen Muskelanspannung bei den betroffenen Kindern kommt; ein Effekt, der z. B. bei depressiven Erkrankungen wohl bekannt ist. Diese vermehrte Muskelanspannung könnte dann zu den in unserer Gruppe gefundenen segmentalen Störungen führen. Es ist wahrscheinlich, dass ein bestimmter Teil der „reversiblen artikulären (segmentalen) Dysfunktionen“ (Baumgartner et al. 1993) muskulär bedingt ist (Goldmann et al. 1997; Buchmann et al. 1998). Für einen so postulierten unspezifischen Effekt einer psychischen Erkrankung auf muskuläre Spannungsverhältnisse spricht die recht hohe Rate der Funktionsstörungen ( 50\% der Kinder waren betroffen) sowohl in der ADHS als auch in der Kontrollgruppe unserer Untersuchung, welche sich ja auch aus Kindern mit kinderpsychiatrischen Störungen zusammensetzte. Damit wären die in der oben zitierten Untersuchung (Buchmann und Häßler 2004) gefundenen manualmedizinischen Funktionsstörungen nicht mit der Erkrankung „ADHS“ kausal zusammenhängend, sondern mit dem Phänomen einer erhöhten Muskelanspannung unter „psychischen Stressbedingungen“ im weitesten Sinne zu erklären. Da es jedoch - nach derzeitigem Kenntnisstand - keine aussagekräftigen Daten zur Häufigkeit manualmedizinischer Funktionsstörungen im Kindes- und Jugendalter gibt, bleibt diese Überlegung spekulativ.

Die als therapeutische Heilversuche durchgeführten Behandlungen der Kopfgelenke blieben auch ohne Effekt auf die Symptomatik des ADHS. 
Schmerzhafte Funktionsstörungen der Kopfgelenksregion sprachen dagegen auf eine manualmedizinische Behandlung gut an. Deshalb sollten manualmedizinische Funktionsstörungen nach unserer Meinung bei diesen Kindern mitbehandelt werden, wobei kontrollierte Studien zur Effektivität zu fordern sind (Brand, Engelbert et al. 2005). Die manuelle Therapie hat jedoch unseren Ergebnissen zufolge keinen unmittelbaren Einfluss auf die ADHS Symptomatik, sondern sollte in ein multimodales Therapiekonzept eingebunden werden (Coenen 1996; Seifert 1996; Buchmann and Häßler 2004).

Eine individuell an Kind und Familie angepasste Therapie setzt natürlich die genaue Diagnostik der Störung am Kind (Fremdbeurteilungsbögen, testpsychologisch, neurophysiologisch) und der familiären Verhältnisse sowie des Umfeldes (Kindergarten/Schule) voraus. Dies ist durch niedergelassene Kollegen nur teilweise zu leisten und setzt eine vernetzte Struktur von niedergelassenem Hausarzt/Pädiater/Manualmediziner/Kinder- und Jugendneuropsychiater bzw. -psychotherapeut, Leistungsträgern (Krankenkassen), kinder- und jugendpsychiatrischer Akutklinik, stationärer Rehabilitation, Sozialpädagogen, Schule und Jugendamt voraus. Die manualmedizinische Behandlung von funktionellen Störungen des Bewegungssystems dieser Kinder fügt sich in die Therapie komorbider Störungen ein. Die Kompensationsfähigkeit von ADHS Kindern gegenüber Stressoren aller Art ist sehr gering, weshalb unter klinischen Gesichtspunkten die manualmedizinische Behandlung gefundener Störungen des Bewegungssystems für das einzelne betroffene Kind durchaus hilfreich, aber nicht kausal wirksam sein kann.

\section{Literatur}

August, G. I., G. M. Realmuto, et al. (1996). Prevalence of ADHD and comorbid disorders among elementary school children screened for disruptive behaviour. J Abnorm Child Psychol 24(5): 571-95.

Baumgartner, H., J. Dvorak, et al. (1993). Grundbegriffe der Manuellen Medizin. Berlin Heidelberg NewYork London Paris Tokyo HongKong Barcelona Budapest, Springer.

Biedermann, H. (1991). Kopfgelenk-induzierte Symmetriestörungen bei Kleinkindern, Kinderarzt 22: $1475-1482$.

Biedermann, H. (1993). Das KISS-Syndrom der Neugeborenen und Kleinkinder, Manuelle Medizin 31: 97-107.

Biedermann, H. (1995). Pathogenese und Therapie frühkindlicher Symmetriestörungen Teil I, Hautnah Paediatr 7: 4-14.

Biedermann, H. (1995). Pathogenese und Therapie frühkindlicher Symmetriestörungen Teil II, Hautnah Paediatr 7: 84-98.

Biedermann, H. and L. Koch (1996). Zur Differentialdiagnose des KISS-Syndroms, Manuelle Medizin 34: 73-81.

Brand, P. L., R. H. Engelbert, et al. (2005). [Systematic review of the effects of therapy in infants with the KISSsyndrome (kinetic imbalance due to suboccipital strain)]. Ned Tijdschr Geneeskd 149(13): 703-7.

Buchmann, J. and B. Bülow (1983). „Funktionelle Kopfgelenkstörungen bei Neugeborenen im Zusammenhang mit Lagereaktionsverhalten und Tonusasymmetrie." Manuelle Medizin 21: 59-62.

Buchmann, J., B. Bülow, et al. (1992). Asymmetrien in der Kopfgelenksbeweglichkeit von Kindern. Manuelle Medizin 30: 93-95.

Buchmann, I., W. Gierow, et al. (2007). Restoration of disturbed intracortical motor inhibition and facilitation in attention deficit hyperactivity disorder children by methylphenidate. Biol Psychiatry 62(9): 963-9.

Buchmann, I., W. Gierow, et al. (2006). „Modulation of transcallosally mediated motor inhibition in chil- 
dren with attention deficit hyperactivity disorder (ADHD) by medication with methylphenidate (MPH)." Neurosci Lett.

Buchmann, J. and F. Häßler (2004). Aufmerksamkeits-Defizit Hyperaktivitätssyndrom (ADHS) - Manualmedizinische, neurophysiologische und kinderneuropsychiatrische Befunde. Manuelle Medizin 42: 195-202.

Buchmann, I., K. Wende, et al. (1998). Gezielte manualmedizinische Untersuchung der Kopfgelenke vor, während und nach einer Intubationsnarkose mit vollständiger neuromuskulärer Blockade. Man Med 36: $32-36$.

Buchmann, I., K. Wende, et al. (2005). Manual treatment effects to the upper cervical apophysial joints before, during, and after endotracheal anesthesia: a placebo-controlled comparison. Am I Phys Med Rehabil 84(4): 251-7.

Buchmann, J., A. Wolters, et al. (2003). Disturbed transcallosally mediated motor inhibition in children with attention deficit hyperactivity disorder (ADHD). Clin Neurophysiol 114(11): 2036-42.

Coenen, W. (1996). Die sensomotorische Integrationsstörung. Manuelle Medizin 34: 141-145.

Coenen, W. (1996). Manualmedizinische Diagnostik und Therapie bei Säuglingen. Manuelle Medizin 34108-113.

Coenen, W. (2002). „Koordinations- und Konzentrationsstörung im Kindesalter.“ Manuelle Medizin 40: 352-358.

Goldmann, R., A. Bornscheuer, et al. (1997). „Gelenkblockierungen und gestörtes Gelenkspiel unter Muskelrelaxation." Manuelle Medizin 35: 56-58.

Gutmann, G. (1968). Das cervical-dieencephal-statische Syndrom des Kleinkindes." Manuelle Medizin 6: 112-119.

Mau, H. (1962). Die sogenannte Säuglingsskoliose und ihre krankengymnastische Behandlung. Stuttgart, Thieme.

Mau, H. (1979). Zur Ätiopathogenese von Skoliose, Hüftdysplasie und Schiefhals im Säuglingsalter. Z Ortop 117: 784-789.

Moll, G. H., H. Heinrich, et al. (2000). Deficient intracortical inhibition in drug-naive children with attentiondeficit hyperactivity disorder is enhanced by methylphenidate. Neurosci Lett 284(1-2): 121-5.

Riede, D. and R. Tomaschewski (1983). Beitrag zur Ätoplogie der idiopathischen Skoliose nach manualtherapeutischen Gesichtspunkten. Manuelle Medizin 21: 67-70.

Rohde, L. A., J. Biederman, et al. (1999). ADHD in a school sample of Brazilian adolescents: a study of prevalence, comorbid conditions, and impairments. I Am Acad Child Adolesc Psychiatry 38(6): 716-22.

Seifert, I. (1981). Kopfgelenksblockierung bei Neugeborenen. Rehabilitacia suppl. 10: 53-57.

Seifert, I. (1996). Praktische Bemerkungen zur manuellen Behandlung der Schäglagedeformitäten der Säuglinge. Manuelle Medizin 34: 114-115.

Speckmann, E. J. and W. Wittkowski (1997). Das Substrat der „Blockierung“. anuelle Medizin 35: 176-183.

Theiler, R. (1996). Manualmedizin in der pädiatrischen Spezialsprechstunde. Manuelle Medizin 34: 53-54.

Wender, E. H. (2002). Attention-deficit/hyperactivity disorder: is it common? Is it overtreated? Arch Pediatr Adolesc Med 156(3): 209-10. 